\title{
BIO-MEDICAL IMAGE SEGMENTATION USING MARKER CONTROLLED WATERSHED ALGORITHM: A CASE STUDY
}

\author{
Ravi $S^{1}$, A M Khan ${ }^{2}$ \\ ${ }^{1}$ Research Scholar, Department of Electronics, Mangalore University, Mangalagangothri - 574199 \\ ${ }^{2}$ Professor and Chairman, Department of Electronics, Mangalore University, Mangalagangothri - 574199
}

\begin{abstract}
Image segmentation is a process in which the objects of interests are selected and separated from the total image to analyze and extract the required data. It is one of the image processing methods, which is widely open for research. Many algorithms have been proposed till date to improve the quality of segmentation and the best one is yet to design. Watershed algorithm is one such reliable approach to segment homogeneous regions in an image. It is superior to region growing method in terms of number of regions obtained after processing. In this paper a robust approach is discussed to improve, the primary drawback of watershed algorithm, over-segmentation. We discuss morphological marker controlled method and the use of gradient image for watershed algorithm to minimize over-segmentation.
\end{abstract}

Keywords: Watershed Algorithm, Morphological Operation, Marker Controlled Watershed, Morphological Reconstruction, Image Segmentation.

\section{INTRODUCTION}

Image is two-dimensional light intensity function of spatial coordinates. If the intensity function and the spatial coordinates are finite and discretised, the image is said to be digital in nature. Processing of such digital image using a digital computer is called digital image processing[9]. There are three levels of image processing like low-level, mid-level and high level image processing. All these levels are concerned mainly in improving the quality of image for human visualization and to extract more information from the image. Among these, image segmentation is one such method which has been widely explored to enhance the quality of information available in the image[6].

Image segmentation can be done in three distinct ways; based on the intensity values (threshold based method), based on discontinuities in the image (edge based method), based on homogeneous regions present in the image (region based method)[10]. The said methods are the conventional methods used for segmentation. Among these, edge based method and region based methods are widely tested. Edge based method identifies the edges, which are basically sharp intensity transitions, local to a region, in the images and mark the pixel as boundary element. In contrast, region based method groups the pixels which share common characteristics to form homogeneous regions[1]. Region based segmentation can be done in two different ways as growing method and split \& merge method. Region growing method is usually preferred for its reliable and robust output.
Watershed algorithm is hybrid approach of 'good characteristics' of edge based segmentation and region based segmentation[9] and hence gives better result than its parents. In this paper we discuss the theory of watershed transform and apply the algorithm for few test images for better understanding. We also discuss marker controlled approach using morphological operations to improve the quality of watershed algorithm and to overcome its drawback of oversegmentation.

Morphological operations are the mathematical set theory operations that are very simple to use and mainly concerned with the ordering of the elements instead of the numerical values of members of the set[6]. This mathematical morphology when combined with the logical operations gives very good results in image processing for performing operations like blurring, sharpening reconstruction and so on[8]. Morphological operations are very much useful in watershed algorithm to construct dam between neighboring regions which is the key process in watershed algorithm.

The paper is arranged as follows: section II highlights watershed algorithm, section III is on basic morphological operations, in section IV we discuss marker controlled watershed algorithm, in section $\mathrm{V}$ application of our algorithm is shown and in section VI results are discussed.

\section{WATERSHED ALGORITHM}

The watershed algorithm includes the three basic segmentation approaches like threshold based, edge detection and region 
based segmentation[1][2] and hence it provides more stable results than these methods individually. It uses morphological operations as well to set proper boundaries between adjacent regions and morphological operations are discussed in next section.

To understand watershed algorithm it is to be known the following three 'points':[9]

1. Points belonging to regional minimum

2. Point of catchment basin / watershed lines.

3. Points at which water drop can more likely to occupy other than a specific watershed.

The watershed lines are those single minimum values to which a drop of water falls with certainty. The gradient of the image is to be found before applying watershed. The characteristic of a pixel will be compared with the neighboring pixel and if found similar, the pixels are added to form a region. The process is carried out till edge of the region is found or the neighboring regions are above to merge. At this stage a dam is built to avoid the merging of two different regions. As a result, after testing all the pixels only the top of the dam is visible and is the resultant of segmentation of various regions in the test image.

Let $M 1, M 2, \ldots, M R$ sets denoting the coordinates in the regional minima of an image $g(x, y)$, where $g(x, y)$ is the pixel value of coordinate $(x, y)$. Let $C(M i)$ be the coordinates in the catchment basin associated with regional minimum $M i$ and let $T[n]$ be the set of coordinates $(s, t)$ for which $g(s, t)<\mathrm{n}$ and is given by

$$
T[n]=\{(s, t) \mid g(s, t)<n\}
$$

Then the process of watershed algorithm is discussed as below:

\section{Step1:The boundary value of the pixels is to be found and the minimum value is to be noted}

The boundary values of the pixels of $g(x, y)$ is to be found and the minimum value is to be assigned to $\mathrm{M}_{\mathrm{i}}$. Flooding is done by initializing $\mathrm{n}=\min +1$

Let $C_{n}\left(M_{i}\right)$ as the coordinates in the catchment basin associated with minimum $M i$ that are flooded at stage $n$.

Step2:Compute catchment basins.

Compute $\mathrm{C}_{\mathrm{n}}\left(\mathrm{M}_{\mathrm{i}}\right)=\mathrm{C}\left(\mathrm{M}_{\mathrm{i}}\right) \cap \mathrm{T}[\mathrm{n}]$

$=\left\{\begin{array}{cr}1 & \operatorname{Cn}(\mathrm{Mi}) \\ 0 & \text { for }(x, y) \in C(\text { Mi)andalso }(x, y) \text { belongstoT }[n]\end{array}\right.$
Let $C[n]$ denote the union of the flooded catchment basins at stage n:

$$
\mathrm{C}[\mathrm{n}]=\bigcup_{i=1}^{R} \operatorname{Cn}(\mathrm{Mi})
$$

Set $n=n+1$.

\section{Step3:Derive the set of connected components}

Derive the set of connected components in $T[n]$ denoting as $Q$. For each connected component $q \in Q[n]$, there are three conditions:

a. If connected components is empty it represents a new minimum is encountered.

If $q \cap C[n-1]$ is empty, connected component $q$ is incorporated into $C[n-1]$ to form $C[n]$ because it represents a new minimum is encountered.

b. If connected components contains at least one connected component it means connected components lies within the catchment basin of some regional minimum.

If $q \cap C[n-1]$ contains one connected component of $C[n-1]$, connected component $q$ is incorporated into $C[n-1]$ to form $C[n]$ because it means $q$ lies within the catchment basin of some regional minimum.

c. If connected components contains more than one connected component it represents all or part of a ridge separating two or more catchment basins and set them as "dam".

If $q \cap C[n-1]$ contains more than one connected component of $C[n-1]$, it represents all or part of a ridge separating two or more catchment basins is encountered so that we have to find the points of ridge(s) and set them as "dam".

\section{Step4. Construct dam for all possible catchment basins.} Construct $C[n]$ using the values obtained for $\mathrm{C}_{\mathrm{n}}\left(\mathrm{M}_{\mathrm{i}}\right)$ and $\mathrm{c}[\mathrm{n}]$ Set $n=n+1$.

\section{Step5. Repeat Step 3 and 4 until $n$ reaches $\max +1$.}

\section{MORPHOLOGICAL OPERATIONS}

Mathematical morphology is a tool for extracting region shape[3]. It can also be used for pre-processing operations much like filters used in spatial filtering operations. Morphological operations are centered on set theory but, when used along with logical operations it makes it much simpler 
than the conventional methods like convolution used in image processing[6]. It is based on ordering of the pixels instead of the numerical value of pixels. Morphological operations uses binary image of very small size called as structuring elements to process the test image. The structuring element works much like the spatial filters that are used in spatial domain processing[8]. Dilation and Erosion are the two fundamental operations that are used in morphological operations and any other morphological operation, like opening, closing, reconstruction etc., are the combination of these two operations[9].

Dilation: The dilation of an image A by structuring element B is defined as

$$
A \oplus B=\left\{z \mid{\widehat{B})_{z}}_{z} \cap \neq \neq \emptyset\right\}
$$

If set $\mathrm{B}$ is reflected about its origin and shifted by $\mathrm{z}$, then the dilation of $\mathrm{A}$ by $\mathrm{B}$ is the set of all displacements $\mathrm{z}$ such that $\mathrm{B}^{\wedge}$ and $\mathrm{A}$ have at least one common element. The dilation operation makes the boundary of the object to grow, to the extent decided by the shape and size of the structuring element. This effect is analogous to the smoothing operation performed in the spatial low pass filtering application. This operation is used to fill 'holes' in the object of an image. The other consequence of performing the dilation operation is the blurring effect.

Erosion: The erosion of an image A by structuring element B is defined as

$$
A \ominus B=\left\{z \mid(B)_{z} \subseteq A\right\}
$$

The erosion of image A by structuring element $B$ is the set of all points $\mathrm{z}$ such that the structuring element $\mathrm{B}$ is translated by $\mathrm{z}$ is a subset of the image. The erosion operation is contradictory to the dilation operation and it causes the boundary of the object to shrink and is decided by the shape and size of the structuring element. The thinning effect is equivalent to the spatial high pass filtering. The erosion operation removes those structures which are lesser in size than that of the structuring element. It can be used to remove the noisy 'connection' between two objects in the image.

Image reconstruction: Reconstruction is a morphological transformation that involves two images, a marker image and a mask image, and a structuring element.

If $G$ is the mask and $F$ is the marker, the reconstruction of $G$ from $F$, denoted $R_{G}(F)$, is defined by the following iterative procedures:[8]

1. Initialize $h_{1}$ to be the marker image, $F$.

2. Create the structuring element: $B$

3. Repeat:

$$
h_{k+1}=\left(h_{k} \oplus B\right) \cap G
$$

until $h_{k+1}=h_{k}$.

4. $R_{G}(F)=h_{k+1}$.

Marker $F$ must be a subset of $G: F \subseteq G$

\section{MARKER CONTROL METHOD:}

The application of watershed algorithm is greatly influenced by the noise and other local irregularities in the image [5]. This leads to over-segmentation of regions to an extent that the segmented image itself looks like a noisy image most of the times and hence can be useless for diagnosing the abnormality by a doctor. This is the major drawback of watershed algorithm [6] [5][3][9]. To avoid this problem markers are used for segmentation. A marker is a connected component belonging to an image. The markers which are connected components do possess the same intensity values and are treated as regional minima. Markers can be classified as internal (foreground) or external (background) depending on its location from region of interest.

The use of markers gives a priori knowledge about segmentation; this knowledge often consists in the number and the positions of the regions through the definition of some markers, and thus simplifies the problem of oversegmentation. The result of using markers is shown in section VI which clearly shows the improvement of segmentation over conventional watershed method.

\section{IMPLEMENTATION}

We designed the algorithm by combining the watershed algorithm, morphological operations and by providing markers for better segmentation. The difference between the existing approach and ours is that we had used bigger size gradient filters compared to the filters used for conventional watershed algorithm. The methodology followed is given below.

Read the image and convert it into suitable form

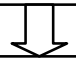

Find out the gradient of the image using a bigger

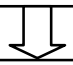

Annlv mornhological image reconstruction

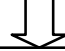

Calculate regional maxima in image

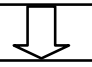

Ohtain foreoround markers \& hackoround

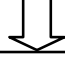

Annlv watershed algorithm to get segmented

Fig-1: Flow chart of the proposed algorithm.

\section{RESULTS AND DISCUSSION}

We had applied the above algorithm on MRI of brain and on mammogram images and compared with the conventional watershed algorithm and the result is given below. The first 
column of image is the original test images; of which two corresponds to MRI of brain and other two are mammogram images. Second column of images is the output of conventional watershed algorithm, third column corresponds to output of the proposed method and fourth column is the result of overlapping third column on the first column. The results shown below are obtained using Matlab2013a with system configuration Inter(R) Pentium(R)4,CPU 2.80GHz, 1GB RAM, 32 bit OS.
It is evident that the proposed algorithm gives better result than the conventional algorithm. The problem of over segmentation is overcome by applying markers on the gradient image. By declaring the internal and external markers we restrict the segmentation to a single object and the background. To carry out this segmentation we can use any of the approaches available but the watershed gives better result.
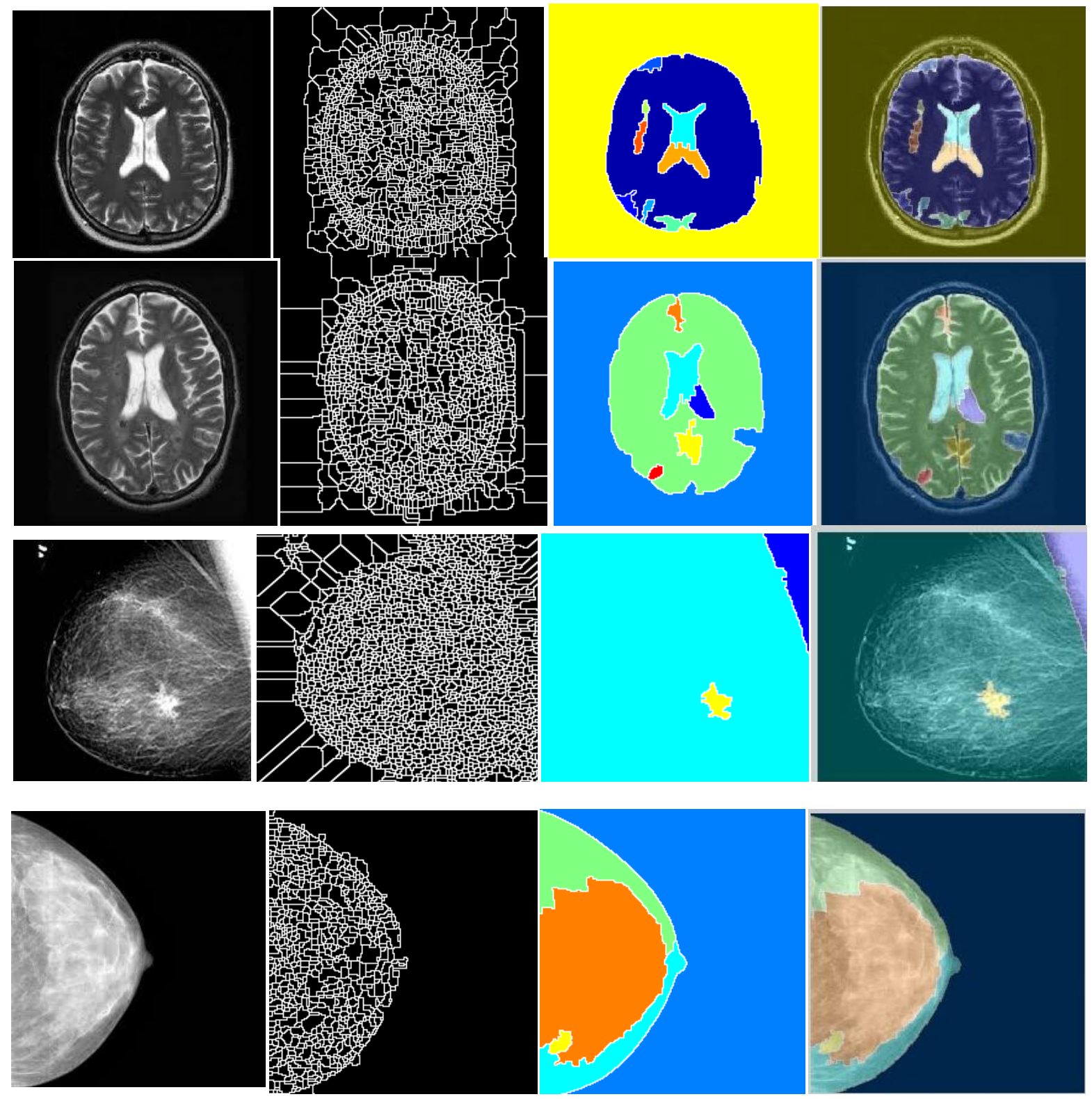

Fig-2 First column: Original images; Second column: Conventional watershed output; Third column: Result of proposed algorithm; Fourth column: Output of proposed algorithm overlapped with original image. 
We conclude that the proposed algorithm is superior to conventional algorithm in terms of over-segmentation. Despite of giving a good output the proposed algorithm in future it can still be improved to get accurate results by using mathematical transformations.

\section{FUTURE WORKS}

We have observed that though there is considerable reduction in the over-segmentation, the obtained image is not ready-touse for diagnosing since it fails to mark the boundary between adjacent regions that vary slightly in the intensities. One of the possible ways to overcome this is by extracting high frequency components in the images to give precise idea about markers. We are working on this to make the processed image ready to use at once the output is obtained.

\section{REFERENCES}

[1] Kostas Haris, Serafim N. Efstratiadis, NicosMaglaveras, and Aggelos K. Katsaggelos, "Hybrid Image Segmentation Using Watersheds and Fast Region Merging", IEEE Transactions On Image Processing, Vol. 7, No. 12, December 1998.

[2] S. Beucher,"'The Watershed Transformation Applied to Image Segmentation", Centre de MorphologieMathematique, Ecolos des Mines de Paris, France.

[3] K. Parvati, B. S. Prakasa Rao and M. Mariya Das Image Segmentation Using Gray-Scale Morphology and Marker-Controlled Watershed Transformation Volume 2008, Article ID 384346, 8 pages, Hindawi Publishing Corporation Discrete Dynamics in Nature and Society.

[4] SébastienLefèvre ,"Knowledge from Markers in Watershed Segmentation" LSIIT, CNRS / University Louis Pasteur - Strasbourg I Parcd'Innovation, Bd Brant, BP 1041367412 IllkirchCedex, France.

[5] Amanpreetkaur, Ashish Verma, "The Marker-Based Watershed Segmentation -A Review" International Journal of Engineering and Innovative Technology (IJEIT), Volume 3, Issue 3, September 2013.

[6] Ravi S and A M Khan, "Morphological Operations for Image Processing: Understanding and its Applications", Proc. 2nd National Conference on VLSI, Signal processing \& Communications NCVSComs-2013, 11 -12th Dec. 2013.

[7] Sameer Ruparelia, Implementation of Watershed Based Image Segmentation Algorithm in FPGA, Master Thesis Nr. 3256, Department of Parallel Systems, Institute of Parallel and Distributed Systems, Universit $\square$ atsstra, Stuttgart.

[8] Marker Controlled Watershed Algorithm IPT, MatLab Documentation.

[9] R.C Gonzalez and R.E. Woods, Chapter 9\& 10; 'Digital Image Processing', third edition, PHI Publications, 2008.
[10] S. Nagabhushana, "Computer Vision and Image Processing”, New Age International Publishers, 2005.

[11] http://www.mathworks.in 